\title{
GPU-ACCELERATED MONTE CARLO CODE FOR FAST DOSE RECALCULATION IN PROTON BEAM THERAPY*
}

\author{
A. Ruciński, J. Gajewski, P. Olko
}

The H. Niewodniczański Institute of Nuclear Physics PAN, Kraków, Poland

\section{RINALDI}

Institute of Nuclear Physics of Lyon, France

V. Patera, A. Schiavi

Sapienza University of Rome, Italy

(Received October 2, 2017)

Proton therapy has rapidly grown in the past thirty years and it has become a superior alternative to conventional radiotherapy for certain clinical indications. Proton therapy offers high dose selectivity due to the protons' distinct depth dose profile which potentially allows to deliver high dose to the tumor while sparing healthy surrounding tissue. Monte Carlo (MC) simulations, which take explicitly into account all the details in the interaction of particles with human tissues, are considered to be the most reliable tool to reproduce the complexity of mixed-field irradiation in a nonhomogeneous environment. The advent of general-purpose programming GPU cards prompted the development of trimmed-down MC-based dose engines, which can significantly reduce the plan recalculation time with respect to standard MC codes on CPU hardware. In this contribution, the GPU-accelerated MC treatment planning system (TPS) Fred developed by the University of Rome is presented (Schiavi et al., Phys. Med. Biol. (2017)). The current status of the implementation in Fred of the experimentally measured physical beam model data used for treatment planning at the Cyclotron Center Bronowice (CCB) Kraków proton beam therapy centre is reported. Three-dimensional dose distributions of proton pencil beams in a water phantom, i.e. lateral profiles and depth dose distributions, are compared for different beam configurations.

DOI:10.5506/APhysPolB.48.1625

* Presented at the $2^{\text {nd }}$ Jagiellonian Symposium on Fundamental and Applied Subatomic Physics, Kraków, Poland, June 3-11, 2017. 


\section{Introduction}

The advantage of Proton Beam Therapy (PBT) compared to X-ray radiation therapy is due to the finite range of protons (Bragg peak). Protons slow down continuously when penetrating the patient body and eventually stop in a certain depth. This allows to treat tumour targets located deep within healthy tissue [1]. The clinical exploitation of these properties of proton beams requires accurate dose calculation methods. In the clinical routine, a treatment planning system (TPS) is used to predict and visualise the therapeutic dose distribution prescribed and delivered to the patient.

Monte Carlo (MC) simulations incorporate detailed knowledge of physics in the patient dose calculation, i.e. particle transport and interactions within a medium, and offer increased accuracy with respect to the analytical TPS used in the clinic. An MC code for recalculation of patient treatment plans can potentially save beam time needed for experimental treatment plan validation and can account for large density gradients and complex geometries in the patient. Prior to use in the clinic, $\mathrm{MC}$ codes must be benchmarked against the PBT centre specific proton beam model [2-4]. The proton beam model is obtained from the commissioning measurements performed during the PBT centre start-up and is implemented in the TPS.

Clinical use of general purpose MC codes [5,6] running on computer stations or even computer clusters is demanding due to the long computation time needed to calculate the patient dose distribution. The adaptation of MC codes to general purpose Graphical Processing Units (GPU cards) would reduce computation time. This triggered the development of in-house trimmed-down MC codes dedicated to PBT dose computations. GPU-accelerated MC codes can reduce the computation time for dose distribution calculations from several hours to a few minutes and make the use of MC Treatment Planning Systems (MC-TPS) in the clinical routine possible.

The software platform Fred is an MC code specifically developed for PBT dose calculations on GPU [7]. Fred is currently being commissioned against the beam model of the CCB Kraków PBT centre. This proceeding reports on the comparison of single beam dose distributions computed with Fred and with Eclipse TPS used in CCB Kraków for patient treatment.

Fred: Fast paRticle thErapy Dose evaluator. The software toolkit Fred was developed at the University of Rome to reduce dose recalculation time in PBT by GPU hardware. The parallelization capability of GPUs can significantly reduce the computation time up to a factor 100 with respect to a standard software running on CPU. The tracing kernel reaches tracking rate of 5 million primary/s on a single GPU card [7]. The transport of proton beams in the patient is accurately described through MC methods. Fred 
uses the anatomical information from Computed Tomography (CT) images to build a voxelised geometry of the patient containing the atomic tissue composition. Fred can be used as an efficient tool for dose recalculation on the day of the treatment and/or to support experimental verification of the treatment plan. Fred is also capable of calculating the tracks of secondary protons and reconstruct secondary proton emission profiles.

CCB Kraków proton beam therapy centre is in clinical operation since 2010 offering ocular cancer treatment. Since October 2016, proton therapy was moved to the new facility. An IBA Proteus C-235 cyclotron delivers the proton beam to the eye treatment room, to two rotational gantries equipped with pencil beam scanning, and to the experimental hall. Until now (July 2017), about 50 head and neck cancer patients were treated with gantry and more than 150 ocular cancer patients were treated in eye treatment rooms. Dedicated Quality Assurance (QA) protocols were developed by a team of about 20 physicists responsible for the safety of patient treatments. The previous cyclotron, used before 2016 for eye treatments, now provides a $62 \mathrm{MeV}$ proton beam for research purposes $24 / 7$.

\section{Fred MC-TPS commissioning in Kraków PBT centre}

The key element of Fred commissioning against the beam model of the CCB Kraków PBT centre is the comparison of single beam dose distributions computed with Fred and with Eclipse TPS. As an example, we report dose calculations for the nominal TPS proton beam energies of 100 and $150 \mathrm{MeV}$. The proton pencil beam was simulated starting at a virtual source $1 \mathrm{~m}$ upstream from isocentre plane and impinging isocentrically onto a water phantom of $12 \times 12 \mathrm{~cm}$ lateral dimensions and $40 \mathrm{~cm}$ length, as illustrated in Fig. 1. The results of the simulations were stored in a 3D dose matrix of the same dimensions as the water phantom and analysed using in-house developed Matlab software tools.

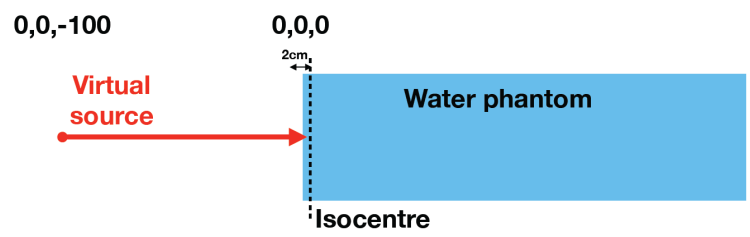

Fig. 1. Water phantom simulation setup used for the dose computations with Eclipse TPS and Fred MC-TPS.

Figure 2 (first row) shows a 2D profile in the isocentre plane simulated with Fred using $10^{9}$ primary protons. Integrated depth dose (IDD) profiles obtained with Fred and the Eclipse TPS are compared in Fig. 2 (second 
row). The beam range (maximum of the Bragg peak: $R_{\max }$ ) obtained from the fit of an analytical approximation of the Bragg curve to the IDD [8, 9]. Agreement of $R_{\max }$ between Fred and TPS simulations was found to be better than $0.1 \mathrm{~mm}$.
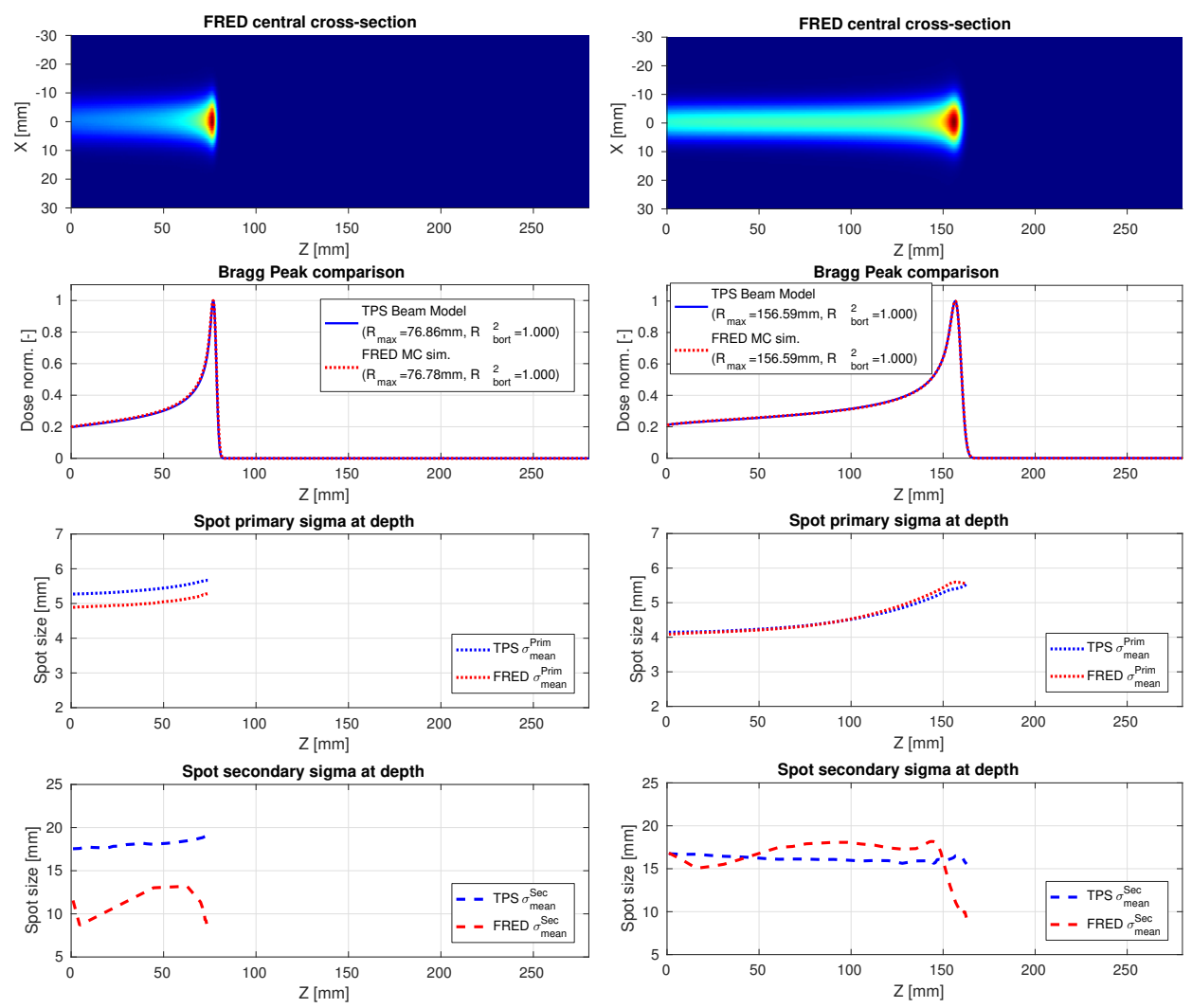

(a) $E=100 \mathrm{MeV}$

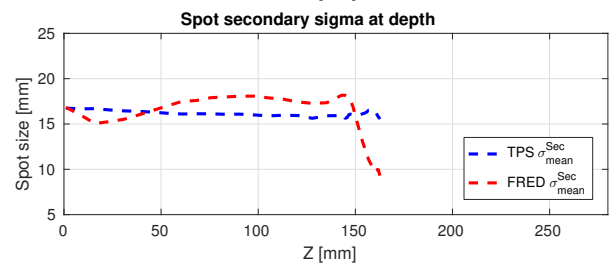

(b) $E=150 \mathrm{MeV}$

Fig. 2. Comparison of depth dose distributions (first and second row) and lateral profile Gaussian fit parameters as a function of depth (third and fourth row) simulated in water for TPS nominal proton beam energy 100 and $150 \mathrm{MeV}$ (left and right column).

Lateral profiles of the beam were fitted at each depth in $0.2 \mathrm{~mm}$ steps with single and double Gaussian functions. An example of the fit performed in the $X$-plane in the depth of the Bragg peak $(z=156.6 \mathrm{~mm})$ for the $150 \mathrm{MeV}$ proton beam is shown in Fig. 3 in logarithmic scale. The contribution of the secondary term in the double Gaussian fit resulting from nuclear interactions is visible. 


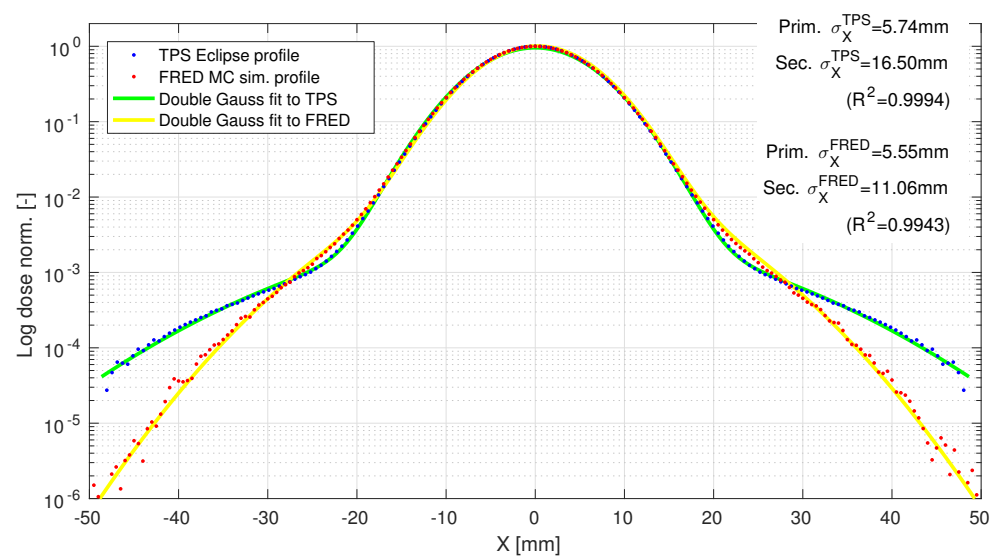

Fig. 3. Lateral profiles of proton beam in water for the TPS nominal beam energy $E=150 \mathrm{MeV}$ fitted at Bragg peak depth $(z=156.6 \mathrm{~mm})$.

The primary and secondary terms of the double Gaussian fit as a function of depth are shown in the third and fourth row of Fig. 2, respectively. Proper tuning of the initial beam parameters (beam size and convergence) in the Fred MC code will be required to obtain satisfying modelling of the low dose envelope (secondary Gaussian). Further studies of the primary and secondary Gaussian terms characteristics will include modelling of the beam scattering in nozzle in Fred, which is expected to compensate for the observed deviations. This is essential to guarantee a good agreement between patient dose distribution calculations obtained with Fred, Eclipse, and treatment plan dose verification measurements (patient QA data) [10, 11].

\section{Conclusions}

Accuracy and time performance of the Fred MC-TPS code exploiting GPU hardware is promising for its application in the proton beam therapy. Preliminary results of the comparison of the 3D dose distributions of single pencil beams computed in water show a good agreement between Fred MC and Eclipse TPS. Fred MC code is intended to support patient dose calculations and QA in CCB Kraków proton beam therapy centre.

This research was supported by the Foundation for Polish Science (FNP) in the frame of Reintegration programme (POWROTY/2016-2/6). This research was supported in part by the PL-Grid Infrastructure. 


\section{REFERENCES}

[1] J.S. Loeffler, M. Durante, Nat. Rev. Clin. Oncol. 10, 411 (2013).

[2] K. Parodi et al., Phys. Med. Biol. 57, 3759 (2012).

[3] C. Grassberger et al., Phys. Med. Biol. 60, 633 (2015).

[4] F. Fracchiolla et al., Phys. Med. Biol. 60, 8601 (2015).

[5] A. Ferrari et al., FLUKA: A Multi-particle Transport Code (program version 2005), Technical Report CERN-2005-10, INFN/TC 05/11, SLAC-R-773, Geneva 2005.

[6] S. Agostinelli et al., Nucl. Instrum. Methods Phys. Res. A 506, 250 (2003).

[7] A. Schiavi et al., Phys. Med. Biol. 62, 7482 (2017).

[8] T. Bortfeld, Med. Phys. 24, 2024 (1997).

[9] J. Gajewski, Bragg Peak Analysis, MATLAB Central File Exchange, 2017.

[10] X. Zhu et al., Med. Phys. 40, 041723 (2013).

[11] J. Shen et al., Med. Phys. 43, 6544 (2016). 